\title{
Vitamin E-Coated Dialyzer Inhibits Oxidative Stress
}

\author{
Shiho Yamadera ${ }^{a}$ Yuya Nakamura $^{\text {b, c }}$ Masahiro Inagaki ${ }^{d}$ Isao Ohsawa ${ }^{c}$ \\ Hiromichi Gotoh $^{c}$ Yoshikazu Goto ${ }^{c}$ Naoki Sato ${ }^{b}$ Tatsunori Oguchi ${ }^{b}$ \\ Yurika Gomi $^{\text {b }}$ Mayumi Tsuji ${ }^{b}$ Yuji Kiuchi ${ }^{\text {b }}$ Shinichi Iwai ${ }^{\mathrm{a}}$ \\ a Department of Healthcare and Regulatory Sciences, School of Pharmaceuticy, Showa University, Shinagawa-ku, \\ and ${ }^{\mathrm{b}}$ Department of Pharmacology, School of Medicine, Showa University, Shinagawa-ku, Tokyo, 'Saiyu Soka \\ Hospital, Soka City, Saitama-ken, and d Department of Chemistry, College of Arts and Sciences, Showa University, \\ Fujiyoshida City, Yamanashi-ken, Japan
}

\section{Keywords}

Vitamin E-coated dialyzer · Synthetic polymer membrane $\cdot$ Anti-oxidative stress effect $\cdot$ Intracellular reactive oxygen species production - Malondialdehyde . $\mathrm{Cu} / \mathrm{Zn}$-superoxide dismutase

\begin{abstract}
Aim: To examine the effects of vitamin E-coated dialyzer on oxidative stress in vitro. Methods: A dialyzer with a synthetic polymer membrane (APS-11SA) and vitamin E-coated dialyzer (VPS-11SA) were connected to a blood tubing line, and U937 cells were circulated in the device. The circulating fluid was collected at 1, 2, 5, 10, 25, and 50 cycles, which are estimated numbers of passes through the dialyzer. Intracellular reactive oxygen species (ROS) production, malondialdehyde (MDA), and $\mathrm{Cu} / \mathrm{Zn}$-superoxide dismutase (SOD) were quantified. Results: Intracellular ROS production was increased in the first cycle by APS-11SA and was decreased throughout the experiment by VPS-11SA. Intracellular ROS production in the VPS-11SA device was lower, and MDA levels were decreased. MDA levels were lower during VPS-11SA processing
\end{abstract}

than during APS-11SA processing. Cu/Zn-SOD levels remained unchanged. Conclusion: Our results highlight antioxidative-stress effects of a vitamin E-coated dialyzer.

(c) 2017 S. Karger AG, Basel

\section{Introduction}

Many studies have shown anti-oxidative effects of a vitamin E-coated dialyzer in vivo $[1,2]$. Nonetheless, in vitro studies are rare [3], and almost all such studies have involved mini-module dialysis membranes [4]. The dialyzer membrane strongly affects experimental cells, and its effect is large compared with the effects of actual clinical hemodialysis treatment. Thus, the following experimental conditions should be considered to avoid overestimating the effect of the dialyzer membrane: (1) the dialyzer membrane should be what is widely used in actual clinical hemodialysis treatments, and not a mini-module; (2) the number of experimental cells should be the same as the number of lymphocytes in humans; (3) the liquid flow rate and experiment duration should also approxi-

\section{KARGER}

(c) 2017 S. Karger AG, Basel

E-Mail karger@karger.com

www.karger.com/bpu
Yuya Nakamura, MD, PhD

Department of Pharmacology

School of Medicine, Showa University

1-5-8 Hatanodai, Shinagawa-ku, Tokyo (Japan)

E-Maily.nakamura@med.showa-u.ac.jp 
mate the actual clinical hemodialysis treatment. There are no studies on the effects of a vitamin E-coated dialyzer under such experimental conditions. Moreover, monocytes play a central role in atherosclerosis, but there are almost no in vitro studies on the effects of vitamin E-coated dialyzer membranes on monocytes.

We examined the effects of a vitamin E-coated dialyzer on oxidative stress in a simple experimental model (in human monocytic U937 cells) that is closer to actual clinical hemodialysis than a mini-module is.

\section{Methods}

\section{Study Materials and Cell Culture}

Human U937 monocytes (EC85011440) were purchased from the European Collection of Animal Cell Culture (ECACC, UK). All the following operations were carried out under sterile conditions. The cells were cultured by routine methods in Roswell Park Memorial Institute medium (RPMI 1640) containing $11.1 \mathrm{mM} \mathrm{D}$ glucose with $10 \%$ of fetal bovine serum, $100 \mathrm{U} / \mathrm{mL}$ sodium penicillin $\mathrm{G}$, and $100 \mu \mathrm{g} / \mathrm{mL}$ streptomycin sulfate, at $37^{\circ} \mathrm{C}$ and $5 \% \mathrm{CO}_{2}$ for 3-5 days. Subsequently, the cells were diluted to $3 \times 10^{6} / \mathrm{mL}$ and were used in the experiment. The following reagents were also used in the study: fetal bovine serum (Sigma Aldrich, St. Louis, MO, USA); penicillin G sodium (Invitrogen, Carlsbad, CA, USA); streptomycin sulfate (Invitrogen); and 5-(and-6)-chloromethyl-2', and $7^{\prime}$-dichlorodihydrofluorescein diacetate (CM-H2DCF-DA; Molecular Probes, Eugene, OR, USA).

\section{Experimental Equipment}

After we connected the blood tubing line (Kawasumi Blood Tubing Line For Hemodialysis, Kawasumi Laboratories, Inc.) to a synthetic polymer membrane (APS-11SA, membrane area $1.1 \mathrm{~m}^{2}$, Asahi Kasei Medical Co., Ltd.) or a vitamin E-coated dialyzer (VPS$11 \mathrm{HA}$, membrane area $1.1 \mathrm{~m}^{2}$, Asahi Kasei Medical Co., Ltd.), it was fixed to a dialysis machine (Nikkiso Co., Ltd.). Next, after we charged the circuit with $1,000 \mathrm{~mL}$ of an isotonic sodium chloride solution (Kawasumi Laboratories, Inc.), this solution was drained, and the circuit was filled with $250 \mathrm{~mL}$ of RPMI 1640. Subsequently, the circuit was connected to a Magnus tube filled with $250 \mathrm{~mL}$ of RPMI 1640, and the total liquid volume of the culture solution in the circuit was $500 \mathrm{~mL}$ (Fig. 1). Finally, the cells were introduced into the circuit (concentration $3 \times 10^{5} / \mathrm{mL}$ ). Oxygen at $1 \mathrm{~L} / \mathrm{h}$ was continuously administered into the circuit (the inside part at $37^{\circ} \mathrm{C}$ ).

\section{Liquid Flow Rate in the Circuit}

This rate was $200 \mathrm{~mL} / \mathrm{min}$. U937 cells and RPMI 1640 were also circulated, and the circulating fluid was collected from the circuit in 2-mL samples for measurement at 1,2,5,10,25, and 50 cycles, which are the estimated numbers of passes through the dialysis membrane.

\section{Evaluation of Cell Numbers}

These numbers were assessed on a $\mathrm{TC}^{20} \mathrm{TM}$ Automated Cell Counter (Bio-Rad Laboratories, Inc., CA, USA). To determine cell viability, we mixed the trypan blue dye and cell suspension (1:1) in a micro test tube, or we combined $10 \mu \mathrm{L}$ of each on Parafilm. The mixture was pipetted up and down gently 10 times to mix it well. We

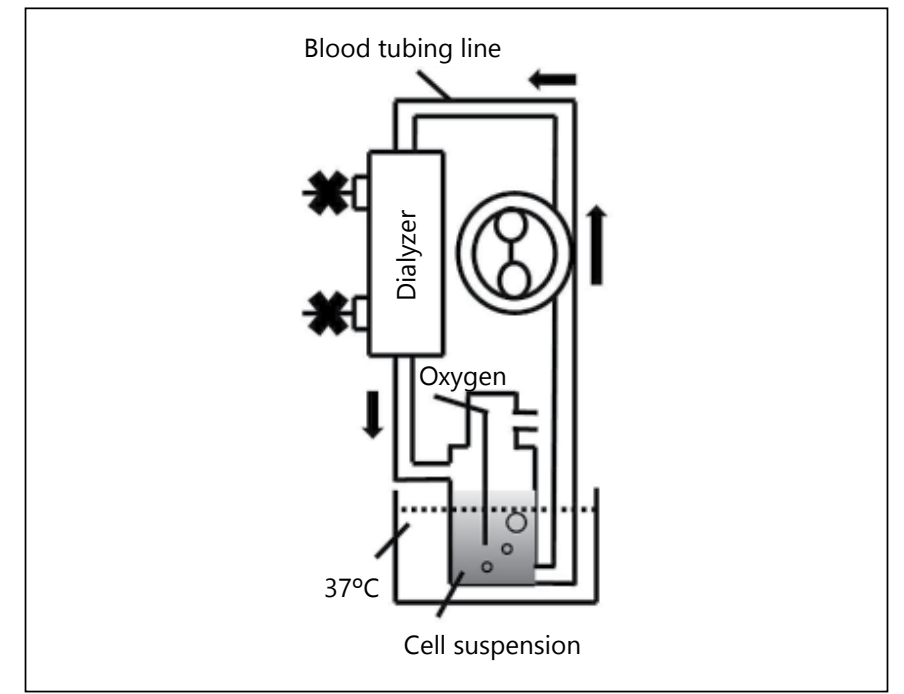

Fig. 1. The experimental device. After we connected the blood tubing line to the dialyzer, it was fixed to the dialysis machine. The total liquid volume of the culture solution in the circuit was 500 $\mathrm{mL}$. The cells were introduced, and the cell concentration was $3 \times$ $10^{5} / \mathrm{mL}$. Oxygen at $1 \mathrm{~L} / \mathrm{h}$ was continuously administered into the circuit (inside part at $37^{\circ} \mathrm{C}$ ).

pipetted $10 \mu \mathrm{L}$ of the mixture into the opening of either chamber on a counting slide. Inserting the counting slide into the slide slot of the $\mathrm{TC}^{20}$ cell counter automatically initiated a cell count: the total cell count, live cell count, and the percentage of live cells were outputted.

\section{Quantification of Reactive Oxygen Species}

CM-H2DCFDA was used to evaluate intracellular reactive oxygen species (ROS) formation in U937 cells. After cellular uptake, CM-H2DCFDA was first modified to $2^{\prime}, 7^{\prime}$-dichlorofluorescein by intracellular esterases, and then by intracellular ROS it is transformed into oxidized fluorescent $2^{\prime}, 7^{\prime}$-dichlorofluorescein. Then we dissolved CM-H2DCFDA in dimethyl sulfoxide, $7 \mu \mathrm{M}$ CM-H2DCFDA was added to the cells and incubated for $15 \mathrm{~min}$ at $37^{\circ} \mathrm{C}$ and $5 \% \mathrm{CO}_{2}$. Subsequently, the cells were washed with $37^{\circ} \mathrm{C}$ heated phosphate-buffered saline. Fluorescence intensity was measured on a plate reader (Berthold Technologies, Bad Wildbad, Germany; excitation and emission at 488 and $525 \mathrm{~nm}$, respectively).

\section{Quantification of Malondialdehyde}

The fraction of U937 cells set aside for lysis was washed to remove protein and other constituents of the medium, and then lysed by sonication. Cell debris were removed by centrifugation at $3000 \times g$ for $10 \mathrm{~min}$ at $4^{\circ} \mathrm{C}$. Malondialdehyde (MDA) was quantified with the BIOXYTECH MDA-586 ${ }^{\mathrm{TM}}$ Kit (OxisResearch $^{\mathrm{TM}}$, Portland, OR, USA). The MDA-586 method is designed to assay free MDA or, after a hydrolysis step, total MDA. The MDA-586 method is based on the reaction of a chromogenic reagent, $\mathrm{N}$ methyl-2-phenylindole, with MDA at $45^{\circ} \mathrm{C}$. One molecule of $\mathrm{N}$ methyl-2-phenylindole yields a stable carbocyanine dye. The MDA-586 method is specific for MDA because 4-hydroxyalkenals do not produce significant color at $586 \mathrm{~nm}$ under the assay condi- 
tions. The colorimetric detection on an enzyme-linked immunosorbent assay (ELISA) plate reader was conducted at $586 \mathrm{~nm}$ (Spectramax, CA, USA).

\section{Assay for Cu/Zn-Superoxide Dismutase}

The lysate fraction of U937 cells was washed to remove protein and other constituents of the medium and then lysed by sonication. Cell debris were removed by centrifugation at $3000 \times g$ for 10 min at $4^{\circ} \mathrm{C}$. $\mathrm{Cu} / \mathrm{Zn}$-superoxide dismutase (SOD) was quantified by an ELISA using a monoclonal antibody $(\mathrm{Cu} / \mathrm{Zn}$-SOD ELISA System; Amersham Pharmacia Biotec Co., Tokyo, Japan). An anti-human $\mathrm{Cu} / \mathrm{Zn}$-SOD antibody was adsorbed onto microwells. $\mathrm{Cu} / \mathrm{Zn}-\mathrm{SOD}$ in the sample or a standard bound to the antibodies adsorbed on the microwells after the first incubation. After a wash, a horseradish peroxidase (HRP)-conjugated anti$\mathrm{Cu} / \mathrm{Zn}$-SOD antibody was added, which binds to $\mathrm{Cu} / \mathrm{Zn}-\mathrm{SOD}$ captured by the primary antibody. After incubation, the unbound HRP-conjugated anti-Cu/Zn SOD antibody was removed during a washing step, and a substrate solution for HRP was added into the wells. The color intensity was proportional to the standard or $\mathrm{Cu} / \mathrm{Zn}$-SOD present in the sample. The acid stop solution was used to stop the color development reaction, and the color intensity was measured on a microplate reader at $450 \mathrm{~nm}$ (SpectraMax).

\section{Statistical Analysis}

Results on cell numbers, intracellular ROS production, MDA, and $\mathrm{Cu} / \mathrm{Zn}$-SOD after the experiment are shown as a percentage of control as compared with the data before the experiment. The results are expressed as mean \pm SEM. The effects of various treatments were compared with those of the no-treatment group by one-way analysis of variance with Bonferroni post-hoc testing. A one-sided $p$ value of $<0.05$ was assumed to indicate statistical significance.

\section{Results}

Cell Numbers, Intracellular ROS Production, MDA

Levels, and $\mathrm{Cu} / \mathrm{Zn}$-SOD Levels at the Start of the

Experiment

The concentration of U937 cells before the experiment was $2.56 \pm 0.22 \times 10^{5} / \mathrm{mL}$. Intracellular ROS production was $68.63 \pm 7.10$ fluorescence intensity $\mathrm{mL} / 10^{5}$ cells, MDA levels were $1.38 \pm 0.10 \mu \mathrm{M} / 10^{5}$ cells, and $\mathrm{Cu} / \mathrm{Zn}$ SOD levels were $0.76 \pm 0.13 \mathrm{ng} / 10^{5}$ cells (Table 1 ).

\section{Number of Live Cells}

This parameter did not change during the experiment (Fig. 2).

\section{Intracellular ROS Production}

This parameter was significantly increased by APS-11SA in the first cycle after the experiment $(p<0.05)$ and continued to increase until cycle 50 (no significance). By contrast, intracellular ROS production in the VPS-11SA device significantly decreased in the first cycle

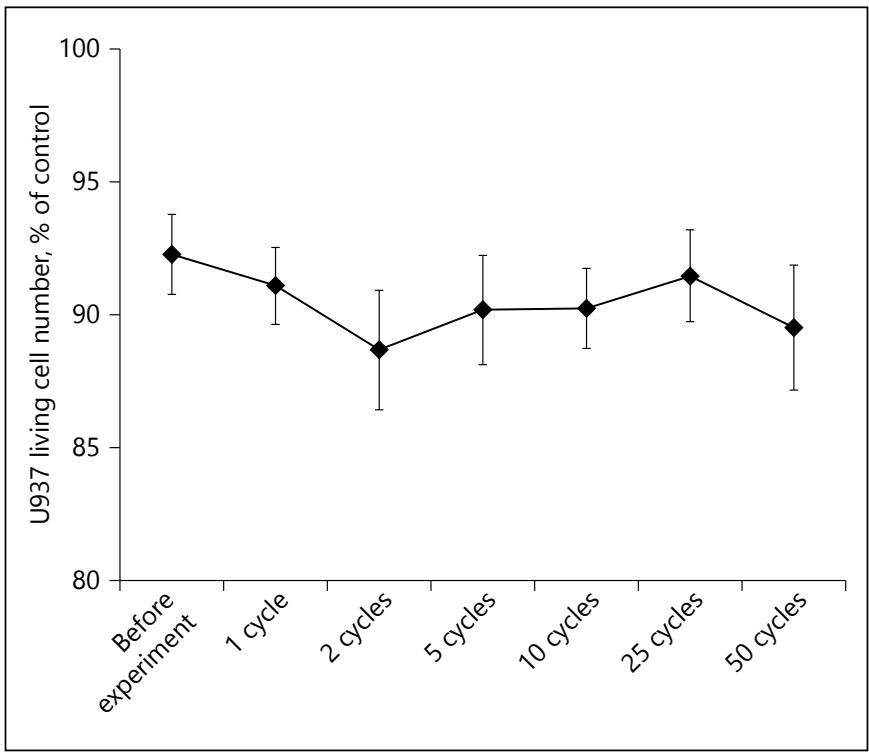

Fig. 2. Number of live cells. These numbers did not change throughout the experiment.

Table 1. Each parameter at the start of the experiment
Live cell number, $10^{5}$ cells $/ \mathrm{mL}$

ROS, FI mL per $10^{5}$ cells

MDA, $\mu \mathrm{M}$ per $10^{5}$ cells

$\mathrm{Cu} / \mathrm{Zn}-\mathrm{SOD}$, ng per $10^{5}$ cells
$2.56 \pm 0.22$

$68.63 \pm 7.10$

$1.38 \pm 0.10$

$0.76 \pm 0.13$
ROS, reactive oxygen species; FI, fluorescence intensity; MDA, malondialdehyde; SOD, superoxide dismutase.

after the experiment $(p<0.05)$ and continued to decrease until cycle $50(p<0.05)$. Intracellular ROS production was significantly lower in the VPS-11SA device (vs. APS$11 S A$ ) at 1,5 , and 25 cycles ( $p<0.05$; Fig. 3 ).

\section{Malondialdehyde}

MDA levels during processing in APS-11SA did not change throughout the experiment, but during processing in VPS-11SA, they significantly decreased in the first cycle after the experiment $(p<0.05)$, and significantly continued to decrease after 10,25 , and 50 cycles $(p<$ $0.01)$. After 10, 25, and 50 cycles, MDA levels were significantly lower in the VPS-11SA device ( $p<0.05$; Fig. 4 ).

\section{$\mathrm{Cu} / \mathrm{Zn}-\mathrm{SOD}$}

$\mathrm{Cu} / \mathrm{Zn}$-SOD levels were not changed by either APS-11SA or VPS-11SA throughout the experiment. There was no difference in $\mathrm{Cu} / \mathrm{Zn}$-SOD levels between the 2 types of samples (Fig. 5). 


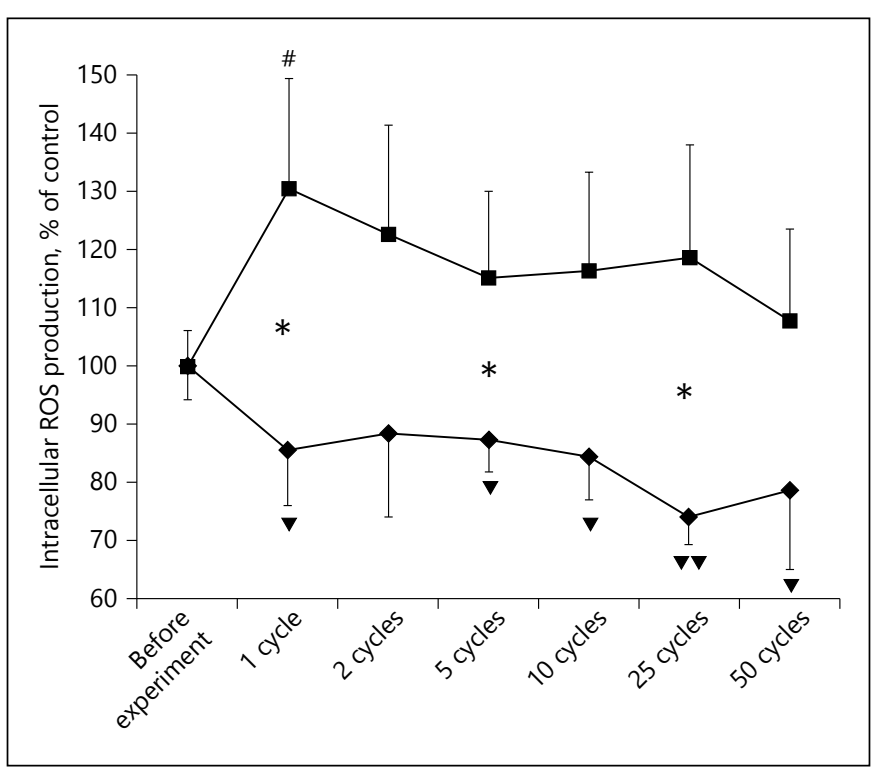

Fig. 3. Intracellular ROS production. This production during processing by the synthetic polymer membrane (APS-11SA) significantly increased in the first cycle of the experiment. Intracellular ROS production during processing by the vitamin E-coated dialyzer (VPS-11SA) significantly decreased in the first cycle of the experiment and continued to decrease until cycle 50. Intracellular ROS production was significantly lower under the influence of VPS-11SA (vs. APS-11SA) after 1, 5, and 25 cycles. APS-11SA; $\checkmark$ VPS-11SA. ${ }^{\#} p<0.05$ vs. before the experiment; ${ }^{\nabla} p<0.05$ vs. before the experiment; ${ }^{\mathbf{\nabla}} p<0.01$ vs. before the experiment; ${ }^{*} p<$ 0.05 VPS-11SA vs. APS-11SA.

\section{Discussion}

Many studies have shown the antioxidant effects of a vitamin E-coated dialyzer in vivo $[1,2]$. In our vitro study, a vitamin E-coated dialyzer decreased intracellular ROS production and MDA levels; therefore, we confirmed the effects of the vitamin E-coated dialyzer in vitro. Vitamin $\mathrm{E}$, one of the radical-scavenging antioxidants and a lipid-soluble vitamin, quickly eliminates and stabilizes active oxygen and free radicals $[5,6]$. Vitamin E present in low-density lipoprotein (LDL) inhibits oxidation of LDL [7]. Our results revealed a decrease in MDA levels in the membrane of U-937 monocytes. MDA is the marker of lipid peroxidation (inhibited by vitamin E). We confirmed the mechanism of antioxidant action via a reduction of lipid peroxidation by vitamin E.

There are a few studies that examined the antioxidative effects of a vitamin E-coated dialyzer in vitro. Almost all in vitro studies involved mini-module dialysis membranes [4]. The dialyzer membrane strongly affected ex-

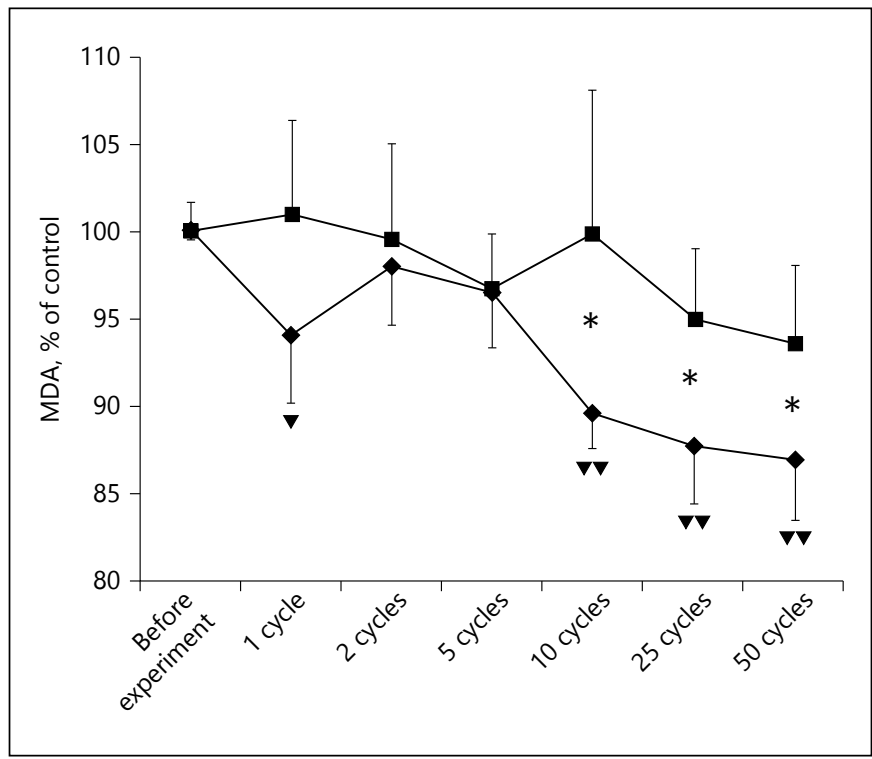

Fig. 4. MDA levels. During processing by APS-11SA, this parameter did not change throughout the experiment. MDA levels under the influence of VPS-11SA were significantly decreased in the first cycle of the experiment and significantly continued to decrease after 10, 25, and 50 cycles. MDA levels affected by VPS-11SA were significantly lower (vs. APS-11SA) after 10, 25, and 50 cycles. APS-11SA; VPS-11SA. ${ }^{\nabla} p<0.05$ vs. before the experiment; $\boldsymbol{\nabla} p<0.01$ vs. before the experiment; ${ }^{*} p<0.05$ VPS-11SA vs. APS-11SA.

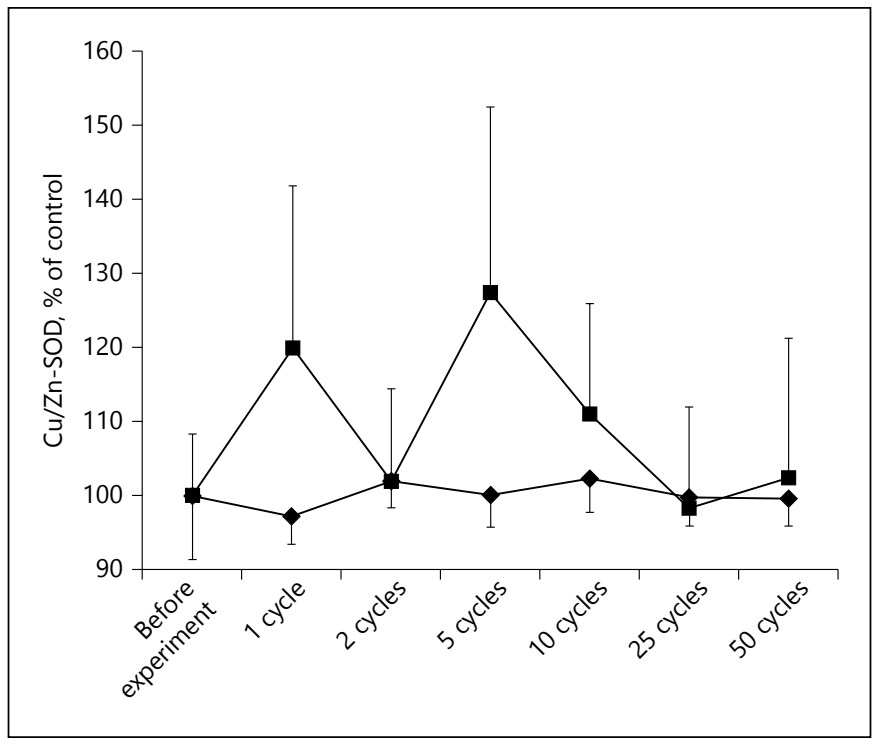

Fig. 5. $\mathrm{Cu} / \mathrm{Zn}$-SOD levels. They were not changed by either APS11SA or VPS-11SA throughout the experiment. There was no difference in $\mathrm{Cu} / \mathrm{Zn}$-SOD levels between samples processed by APS11 SA and VPS-11SA. $\square$ APS-11SA; $\bullet$ VPS-11SA. 
perimental cells, and its effect was large relative to the actual clinical hemodialysis. In this study, we used the following in vitro experimental conditions that are closer to the actual clinical hemodialysis treatment: the same number of U937 cells as human lymphocyte numbers, a hemodialysis membrane that is used in the clinic, 200 $\mathrm{mL} / \mathrm{min}$ liquid flow rate, and 2 -h experiment duration. This was the first in vitro study with an experimental model closer to actual clinical hemodialysis treatment, and our method can evaluate the effects of a vitamin Ecoated dialyzer without overestimating.

Oxidative stress in patients undergoing hemodialysis is high, and the hemodialysis membrane is one of the causes [8]. In the present study, intracellular ROS production increased after processing of cells in a vitamin E-free dialyzer, but MDA levels did not change. ROS levels were highly responsive, reflecting oxidative damage to nucleic acids, proteins, and lipids with the formation of metabolites in vivo. MDA is one of the final products of peroxidation of polyunsaturated fatty acids in the cell. Therefore, our results suggest that the hemodialysis membrane induces oxidative stress unrelated to lipid peroxidation. Some studies revealed that the contact between polynuclear leucocytes and a dialyzer induces oxidative stress $[8,9]$. U937 cells in the present study are monocytes; therefore, the contact between these cells and the dialyzer may also induce oxidative stress.

In in vitro studies, Tsukao et al. [3] reported that the contact with a vitamin E-coated surface reduces platelet activation mediated by superoxide anions. Nevertheless, they evaluated platelet activation and could not assess the effects of vitamin E-coated dialyzer membranes on monocytes. Pertosa et al. [4] reported that vitamin Ecoated dialyzer membranes reduce the activation of Jun $\mathrm{N}$-terminal kinase, which is activated in response to oxidative stress. They used mononuclear cells combined with leukocytes and monocytes, and could not evaluate the effects of vitamin E-coated dialyzer membranes on monocytes alone. Our report is the first in vitro study to do so. Monocytes transform into macrophages in arterial walls and absorb oxidized LDL, forming foam cells via scavenger receptors $[10,11]$. They grow, eventually rupture spilling oxidized materials, and promote atherosclerosis via expansion of the plaque $[12,13]$. Therefore, monocytes play a major role in atherosclerosis. Effective control of oxidative stress and oxidized LDL in the cytosol of monocytes may prevent atherosclerosis. In our study, a vitamin E-coated dialyzer decreased the intracellular ROS production and MDA levels in human monocytic U937 cells. Therefore, from the standpoint of atherosclerosis pathogenesis, we reconfirmed the possibility of prevention of oxidative stress, lipid peroxidation, and atherosclerosis by means of a vitamin E-coated dialyzer.

$\mathrm{Cu} / \mathrm{Zn}-\mathrm{SOD}$, an intracellular antioxidant enzyme and oxidative-stress marker, is downregulated after one hemodialysis treatment session in hemodialysis patients [14]. We hypothesized that $\mathrm{Cu} / \mathrm{Zn}-\mathrm{SOD}$ protein amounts are decreased by the leakage during filtration and via consumption by oxidative stress during hemodialysis. Moreover, decreased production of $\mathrm{Cu} / \mathrm{Zn}-\mathrm{SOD}$ is thought to be due to decreased $\mathrm{Cu} / \mathrm{Zn}$-SOD mRNA expression in leukocytes [14]. In our experimental system without filtration or leakage, $\mathrm{Cu} / \mathrm{Zn}$-SOD levels did not change. Therefore, the leakage with filtration may strongly decrease the amount of $\mathrm{Cu} / \mathrm{Zn}$-SOD during real-world hemodialysis. Further research is necessary.

It is important to evaluate the beneficial effects of longtime use of vitamin E-coated dialyzer membranes, such as in cardiovascular morbidity and mortality, particularly among high-risk patients undergoing dialysis. At present, there are almost no studies about such effects. Because our research is in vitro, the lack of positive clinical results cannot be a limitation of our study. Nevertheless, in vitro research (like our study) closer to actual clinical treatment is likely necessary as a foundation for subsequent clinical studies.

In conclusion, we confirm the useful effects of a vitamin E-coated dialyzer on oxidative stress in a simple experimental model closer to actual clinical hemodialysis (than a mini-module is): human monocytic U937 cells.

\section{Acknowledgement}

S.Y., Y.N., and M.I. devised the study concept and design; S.Y., Y.N., and M.T. collected the data; S.Y. and Y.N. analyzed the data; Y.N. interpreted the data; Y.N. searched the literature; S.Y. and Y.N. drafted the manuscript; Y.N., M.I., I.O., H.G., Y.G., N.S., Y.K., and S.I. made substantial revisions in the manuscript; all authors gave final approval of the manuscript.

\section{Source of Funding}

There are no sources of funding.

\section{Disclosure Statement}

The authors have no conflict of interest to declare. 


\section{References}

1 Kirmizis D, Papagianni A, Efstratiadis G, Memmos D: Impact of inflammation on antioxidative effects of vitamin E-coated membrane dialyzer in patients on chronic hemodialysis. Hemodial Int 2014;18:751-757.

2 Yang SK, Xiao L, Xu B, Xu XX, Liu FY, Sun L: Effects of vitamin E-coated dialyzer on oxidative stress and inflammation status in hemodialysis patients: a systematic review and meta-analysis. Ren Fail 2014;36:722-731.

3 Tsukao H, Kokubo K, Takahashi H, Nagasato M, Endo T, Iizuka N, Shinbo T, Hirose M, Kobayashi H: Activation of platelets upon contact with a vitamin E-coated/non-coated surface. J Artif Organs 2013;16:193-205.

4 Pertosa G, Grandaliano G, Soccio M, Martino C, Gesualdo L, Schena FP: Vitamin E-modified filters modulate Jun $\mathrm{N}$-terminal kinase activation in peripheral blood mononuclear cells. Kidney Int 2002;62:602-610.

5 Packer L, Weber SU, Rimbach G: Molecular aspects of alpha-tocotrienol antioxidant ac- tion and cell signalling. J Nutr 2001;131: 369S-373S

6 Traber MG, Stevens JF: Vitamins C and E: beneficial effects from a mechanistic perspective. Free Radic Biol Med 2011;51:1000-1013.

7 Simon E, Gariepy J, Cogny A, Moatti N, Simon A, Paul JL: Erythrocyte, but not plasma, vitamin $\mathrm{E}$ concentration is associated with carotid intima-media thickening in asymptomatic men at risk for cardiovascular disease. Atherosclerosis 2001;159:193-200.

8 Morena M, Delbosc S, Dupuy AM, Canaud B, Cristol JP: Overproduction of reactive oxygen species in end-stage renal disease patients: a potential component of hemodialysis-associated inflammation. Hemodial Int 2005;9:37-46.

9 Kobari E, Terawaki H, Takahashi Y, Kusano Y, Sakurai K, Matsunaga K, Fukushima N, Suzuki S, Tanaka K, Hayashi Y, Watanabe T, Nakayama M: Dialyzer-related thrombocytopenia due to a polysulfone membrane. Intern Med 2016;55:965-968.
10 Goldstein JL, Ho YK, Basu SK, Brown MS Binding site on macrophages that mediates uptake and degradation of acetylated low density lipoprotein, producing massive cholesterol deposition. Proc Natl Acad Sci U S A 1979;76:333-337.

11 Brown MS, Goldstein JL: Lipoprotein metabolism in the macrophage: implications for cholesterol deposition in atherosclerosis. Annu Rev Biochem 1983;52:223261.

12 Witztum JL, Steinberg D: Role of oxidized low density lipoprotein in atherogenesis. J Clin Invest 1991;88:1785-1792.

13 Ross R: The pathogenesis of atherosclerosis: a perspective for the 1990s. Nature 1993;362: 801-809.

14 Akiyama S, Inagaki M, Tsuji M, Gotoh $\mathrm{H}$, Gotoh T, Gotoh Y, Oguchi K: mRNA study on $\mathrm{Cu} / \mathrm{Zn}$ superoxide dismutase induction by hemodialysis treatment. Nephron Clin Pract 2005;99:c107-c114. 